- Achieving a balancing act between being critical (in an academic way) and constructive (in a practice-oriented way);

- Maintaining your own voice while truthfully reflecting the (often conflicting) voices of multiple stakeholders.

Resolving these tensions may require 'compartmentalisation', whereby two different sets of outputs are produced: for example, a series of high-quality critical journal papers for an academic audience and a series of practice-oriented reports for a practitioner audience. The obvious negative consequences of this approach include the constant need to wear 'multiple hats' and invest extra labour. The biggest difficulty, however, is achieving and maintaining 'embeddedness' in a healthcare organisation, which is a prerequisite for enabling change, without losing critical distance, which is a prerequisite for producing highquality critical qualitative research. Constant self-reflection and peer-debriefing with trusted colleagues are some of the strategies that can alleviate, if not completely resolve, this tension.

\section{BUILDING SUSTAINABLE PARTNERSHIPS WITH LOCAL RESEARCHERS FOR QUALITATIVE HEALTH SYSTEMS RESEARCH}

Amrit Virk, Tim Ensor, William Bolton, David Jayne, Rebecca King. University of Leeds, Leeds, UK

\subsection{6/bmjopen-2019-QHRN.15}

Background International health projects are increasingly engaging local researchers to ensure that interventions are cost-effective and locally relevant. Yet, locating research partners in low and middle-income country settings is not always straightforward particularly when projects are inter-disciplinary, the methods unfamiliar, and the setting, new. Since July 2017, we have initiated a research partnership in North-East India and Sierra Leone to assess surgical need and health system capacity for a global surgery project.

Aim Our presentation will describe our experiences identifying and engaging local stakeholders including government, ethical review bodies, but particularly research teams in India and Sierra Leone to co-design and execute qualitative health research. With a view to inform future global health research, we identify challenges and opportunities for building qualitative health research capacity in both sets of researchers and positioning qualitative research within interdisciplinary health research projects.

Methods Our approach to collaboration for qualitative health research in India and Sierra Leone involves a process of codesigning protocols and ethics applications; jointly producing data collection tools; iterative and cooperative data collection, transcribing and analysis; and co-writing publications and policy briefs.

Expected results This presentation will describe the early stage processes of building local research collaborations for carrying out qualitative health research in low and middle-income countries (LMIC). We identify challenges as well as practical strategies and technological aids to develop cross-country partnerships.

Conclusions While several global health research projects will involve similar processes for building sustainable partnerships with local researchers, less is documented on how early stage collaborations are forged and the challenges overcome particularly in low-resource settings. This presentation contributes to addressing this gap, laying a pathway for future qualitative health research in LMICs.

\section{6}

RARE BUT STILL HEARD: HOW INNOVATIVE METHODS CAN ENHANCE RESEARCH INCLUSION

${ }^{1}$ Kathy McKay, ${ }^{1}$ Veronica Ranieri, ${ }^{2}$ Martine Walmsley, ${ }^{3}$ Douglas Thorburn, ${ }^{1}$ Eilis Kennedy. ${ }^{1}$ Tavistock and Portman NHSFT, London, UK; ${ }^{2}$ PSC Support, Didcot, UK; ${ }^{3}$ Royal Free NHSFT, London, UK

\subsection{6/bmjopen-2019-QHRN.16}

Background Primary Sclerosing Cholangitis (PSC) is a rare, chronic, and incurable liver disease characterised by the inflammation and scarring of the bile duct. The speed and aggression at which the disease can progress may vary between individuals. Both the cause and the underlying triggers for its progression and severity remain unknown. Those living with PSC are more at risk of colorectal and biliary tract cancers, and many will eventually need a liver transplant. As with any transplant, there is uncertainty in waiting for an appropriate donor and people can die in the meantime. Living with such continuing uncertainty can cause enduring and substantial psychological distress. Yet, little research focuses on the mental health and wellbeing of people with PSC and their families.

Objective To understand the experiences of those living with PSC, and their families, in terms of their mental health and wellbeing.

Methods Asynchronous Virtual Focus Groups (AVFGs) were undertaken with people diagnosed with PSC, and their family members. AVFGs were chosen as an appropriate method of data collection for this group as the rarity of the disease meant that participants could not be sampled from close or clustered geographical areas. AVFGs allowed people, across the UK, to participate in this study from their home, without substantial cost or travel.

Results Participants' narratives centred upon their experiences of receiving a diagnosis, contact with health professionals, their support systems and advice they'd give to someone who was newly diagnosed.

Conclusions Uncovering narratives from people affected by rare diseases, such as PSC, has often meant substantial cost or travel for the participant, or their simply not being included due to distance. AVFGs allow for greater inclusion in studies which have the potential to provide positive benefits to the participants.

\section{ENGAGING DISPLACED PEOPLE IN ARTISTIC AND CULTURAL ACTIVITIES USING PARTICIPATORY ACTION RESEARCH}

Linda Thomson. University College London, London, London

\subsection{6/bmjopen-2019-QHRN.17}

Background Many European organisations provide creative activities for displaced people, including refugees and asylum seekers, that aim to reduce social isolation and counter prevalent mental health issues including anxiety, depression, nonaffective psychosis and post-traumatic stress disorder. ${ }^{1-3}$ A growing body of evidence suggests that taking part in artistic and cultural activities benefits human health and wellbeing, ${ }^{4-6}$ though this research has been mostly non-collaborative in nature. Although creative activities are seen to provide major benefits for displaced people, researchers need to 
become increasingly conscious of the value of recording and analysing what has been happening' $\left[{ }^{7} \mathrm{p} 1\right]$ to make effective use of a limited service provision.

Aim The aim was to develop a participatory action research (PAR) method for engaging displaced people to collaboratively assess the full impact of arts and cultural interventions.

Methods A researcher spent five months in a London-based charity conducting a study following PAR principles of observation, focus groups and in-depth semi-structured interviews. Participants acting as 'co-researchers' comprised refugees and asylum seekers $(n=7)$, charity staff $(n=8)$ and volunteers $(n=7)$. Results During focus groups, participants articulated their reflections on the impact of creative activities around three main themes of skills, social engagement, and personal emotions. Interviews based upon these themes showed that artistic and cultural activities impacted positively on participants by helping them to find a voice, create a support network, and learn practical skills useful in the labour market.

Conclusions The study expanded on arts and wellbeing research by exploring the effects of cultural and creative activities on the psychosocial wellbeing of refugees and asylum seekers. By focusing on the relationship between arts, wellbeing and forced displacement, the study was instrumental in actively trying to change the narrative surrounding refugees and asylum seekers often depicted in negative terms in the public sphere. ${ }^{8}$

\section{REFERENCES}

1. Katona C. Non-affective psychosis in refugees. Br Med J 2016;352:11279. doi:10.1136/bmj.i1279.

2. Hollander A, Dal H, Lewis $G$, et al. Refugee migration and risk of schizophrenia and other non-affective psychoses: Cohort study of 1.3 million people in Sweden. Br Med J 2016:352:i1030.https://doi.org/10.1136/bmj.i1030.

3. Robjant K, Robbins I, Senior V. Psychological distress amongst immigration detainees: A cross-sectional questionnaire study. Brit J Clin Psych 2009;48. 275-286. https://doi.org/10.1348/014466508X397007.

4. Camic P, Chatterjee H. Museums and art galleries as partners for public health interventions. Perspect Public Health 2013;133:66-77.

5. Chatterjee H. Museums and art galleries as settings for public health interventions. In S Clift, P Camic (Eds.), Oxford Textbook of Creative Arts, Health, and Wellbeing: International Perspectives on Practice, Policy and Research. New York, Oxford: Oxford University Press 2016; p.281-289.

6. Clift S, Skingley A, Coulton S, Rodriguez J. The effectiveness and costeffectiveness of a participative community singing programme as a health promotion initiative for older people: Protocol for a randomised controlled trial. BMC Public Health 2011;11:1-6. https://doi.org/10.1186/1471-2458-11-142.

7. Kidd B, Zahir S, Khan S. Arts and Refugees: History, Impact and Future. London: Arts Council England, 2008. https://baringfoundation.org.uk/wp content/uploads/ 2014/10/ArtsandRefugees.pdf.

8. Khosravi S. 'Illegal' Traveller: An Auto-ethnography of Borders. Basingstoke: Palgrave MacMillan 2010.

\section{Parallel session - Innovative Research Methods (22 March 14:00 -15:15)}

\section{BREAKING THE GOLD STANDARD: QUALITATIVE METHODS FOR TRIALS OF COMPLEX HEALTH INTERVENTIONS}

Jenevieve Mannell, Katy Davis. University College London, London, UK

10.1136/bmjopen-2019-QHRN.18

Background Randomised controlled trials (RCT) are widely considered to be the 'gold standard' for evaluating health interventions. Qualitative methods are often perceived as an 'add-on' to robust quantitative intervention evaluations.
The current status of qualitative methods within trials undermines the enormous insights qualitative approaches contribute to our understanding of the impact interventions have on human health.

Objectives This presentation examines the 'state of the art' of qualitative methods currently used within trials of complex health interventions.

Methods In early 2018, we completed a rapid review of innovative qualitative methods mentioned in the RCT protocols of complex health interventions for a commentary to a special issue of Qualitative Health Research. 'Innovative' was used to refer to qualitative methods beyond atheoretical interviews or focus groups with trial participants. We generated a list of 189 qualitative research methods from a search of 27 qualitative methodology journals using the terms "innovat" new novel emerg", and then used this list as key terms to search protocols published since 2012 and registered with the ISRCTN trial database.

Results Our search showed that while 1452 of the registered trial protocols mentioned some form of qualitative research, only 34 of these discussed more innovative qualitative methods. Qualitative methods were most often employed during trial process evaluations or as formative research in the pretrial phase. Protocols rarely went into depth about the details of the methods used or explained how their analysis would contribute to the trial results.

Conclusions These gaps significantly undermine the potential of qualitative methods to improve understandings of the successes and failures of interventions in different contexts. We will discuss these gaps and their implications for health intervention evaluation in detail, and also point to some areas of good practice arising from the special issue that we hope will push the field forward.

\section{REFLECTIONS ON DYADIC RESEARCH IN HEALTHCARE}

${ }^{1}$ Angela Sweeney, ${ }^{1}$ Steve Gillard, ${ }^{2}$ Billie Lever-Taylor, ${ }^{2}$ Sonia Johnson. ${ }^{1}$ St George's University of London, London, UK; ${ }^{2}$ UCL, London, UK

\subsection{6/bmjopen-2019-QHRN.19}

Background Although healthcare provision typically occurs within the context of clinician/service user relationships, and there is increasing recognition of the role and importance of families, qualitative data collection typically focuses on individuals (e.g. through interviews) rather than dyads. Dyadic interviewing has a long history in social research, and is now being used in health research to enable a unique exploration of healthcare experiences and relationships.

Aims To reflect on the use of dyadic interviewing in two studies, commenting on utility and potential pitfalls.

Methods The two studies will be described: both employed separate, confidential interviews, one with clinicians and service users on talking therapy assessments, the other with perinatal women and a family member on experiences of mental health services. Responses were compared and contrasted through a focus on 'versus' and 'consensus' coding. This meant that the integrity of individual accounts was retained, with an analysis of convergence and divergence within and between dyads generating a third, etic perspective. ${ }^{1}$

Results Dyadic interviewing in healthcare raises a number of practical and ethical issues: 1) recruitment can be slow, for example because clinicians need the confidence to consent to 